УДК 517.9

\title{
Two-dimensional Plane Thermocapillary Flow of Two Immiscible Liquids
}

\author{
Elena N. Lemeshkova* \\ Institute of computational modelling SB RAS \\ Akademgorodok, 50/44, Krasnoyarsk, 660036
}

Russia

Received 23.01.2019, received in revised form 29.03.2019, accepted 02.04.2019

The problem of two-dimensional stationary flow of two immiscible liquids in a plane channel with rigid walls is considered. A temperature distribution is specified on one of the walls and another wall is heatinsulated. The interfacial energy change is taken into account on the common interface. The temperature in liquids is distributed according to a quadratic law. It agrees with velocities field of the Hiemenz type. The corresponding conjugate boundary value problem is nonlinear and inverse with respect to pressure gradients along the channel. The Tau method is used for the solution of the problem. Three different solutions are obtained. It is established numerically that obtained solutions converge to the solutions of the slow flow problem with decreasing the Marangoni number. For each of the solutions the characteristic flow structures are constructed.

Keywords: interface, thermocapillary, inverse problem, Tau method. DOI: 10.17516/1997-1397-2019-12-3-310-316.

For many liquids the surface tension is well approximated by the linear function. Then energy equality is simplified and has the form [1]

$$
k_{2} \frac{\partial \theta_{2}}{\partial n}-k_{1} \frac{\partial \theta_{1}}{\partial n}=æ \theta \operatorname{div}_{\Gamma} \mathbf{u}
$$

where $æ=-\partial \sigma / \partial \theta, \sigma(\theta)$ is the surface tension coefficient. In equality (1) $k_{j}$ are coefficients of thermal conductivity and $\theta_{j}$ are temperature of liquids, $j=1,2 ; \theta=\theta_{1}=\theta_{1}$ and $\mathbf{u}=\mathbf{u}_{1}=\mathbf{u}_{2}$ are temperature and velocity vector at the interface $\Gamma, \mathbf{n}$ is the normal to $\Gamma$ directed to the second liquid.

The ratio of right-hand side of equation (1) to the terms in left-hand side of equation (1) is estimated by the parameter $\mathrm{E}=æ \theta^{*} / \mu_{j} k_{j}$ (for the first term it is necessary to assume $j=1$ and for the second term $j=2$ ), $\mu_{j}$ are dynamic viscosities, $\theta^{*}$ is the characteristic temperature on the interface. Parameter $E$ determines the influence of interphase energy on the motion of liquids inside the layers. For ordinary liquids at room temperature this parameter is small. For example, it was obtained in experiments that $\mathrm{E} \sim 5 \cdot 10^{-4}$ for the air - ethyl alcohol system at $\theta^{*}=15^{\circ} \mathrm{C}$. Therefore, the right-hand side of equation (1) is often omitted and we have the equalities of the heat flux across the interface. However, for low-viscosity liquids and some cryogenic liquids (for example,liquid $\mathrm{CO}_{2}$ ) the influence of interfacial energy must be taken into account. It is known that viscosity decreases rapidly with increasing temperature. Calculations of motion of bubbles in various liquids [2] showed that value $\mathrm{E}=O(1)$ is achieved at sufficiently high temperatures. The maximum values of $\mathrm{E}$ are attained near the critical points. So, for water we have $\mathrm{E} \sim 0.02$

*elena_cher@icm.krasn.ru

(c) Siberian Federal University. All rights reserved 
at $\theta=303.15 K ; \mathrm{E} \sim 0.6$ at $\theta=573.15 K ; \mathrm{E} \sim 0.7$ at $\theta=623.15 K$ (critical point for water is $\left.\theta_{\text {кр }}=647.30 \mathrm{~K}\right)$.

In the paper we study a two-dimensional stationary flow of two immiscible liquids with a common interface at which the total energy condition is satisfied. The influence of dimensionless parameters on the structures of emerging flows is studied.

\section{Problem formulation}

To describe a two-layer stationary flow of viscous heat-conducting fluids in layers bounded by solid walls $y=0, y=h$ with a common interface $y=l<h$ we assume the following form of velocity and temperature fields

$$
\begin{gathered}
u_{j}^{1}(x, y)=w_{j}(y) x, \quad u_{j}^{2}(x, y)=v_{j}(y), \\
\theta_{j}(x, y)=a_{j}(y) x^{2}+b_{j}(y)
\end{gathered}
$$

where $0<y<l$ for $j=1, l<y<h$ for $j=2 ; u^{1}, u^{2}$ are velocity vector components, $\theta$ is temperature. Such representation of the velocity field corresponds to the well-known Hiemenz solution [3]. The substitution of expression (2) into the equations of motion and heat transfer results in the nonlinear systems of equations for functions $w_{j}(y), v_{j}(y), a_{j}(y)$ and $b_{j}(y)$

$$
\begin{gathered}
v_{j} w_{j y}+w_{j}^{2}=\nu_{j} w_{j y y}+f_{j}, \quad w_{j}+v_{j y}=0 \\
2 w_{j} a_{j}+v_{j} a_{j y}=\chi_{j} a_{j y y} \\
v_{j} b_{j y}=\chi_{j} b_{j y y}+2 \chi_{j} a_{j}
\end{gathered}
$$

where $\nu_{j}$ are the kinematic viscosities, $\rho_{j}=\mu_{j} / \nu_{j}$ are the densities, $f_{j}$ are the constants. The pressures in liquids are distributed as

$$
\frac{1}{\rho_{j}} p_{j}(x, y)=\nu_{j} v_{j y}-\frac{v_{j}^{2}}{2}-f_{j} \frac{x^{2}}{2}+d_{0 j}, \quad d_{0 j}=\text { const. }
$$

The values of $f_{j}$ characterize pressure gradients along the $x$ axis.

Conditions on the rigid walls are

$$
\begin{array}{cll}
w_{1}(0)=v_{1}(0)=0, & a_{1}(0)=a_{10}, & b_{1}(0)=b_{10}, \\
w_{2}(h)=v_{2}(h)=0, & a_{2}(h)=a_{20}, & b_{2}(h)=b_{20} .
\end{array}
$$

The following relations are satisfied on the interface $y=l$

$$
\begin{aligned}
w_{1}(l)=w_{2}(l), \quad & v_{1}(l)=v_{2}(l)=0, \quad a_{1}(l)=a_{2}(l), \quad b_{1}(l)=b_{2}(l), \\
& \mu_{2} w_{2 y}(l)-\mu_{1} w_{1 y}(l)=-2 æ a_{1}(l), \\
& k_{2} a_{2 y}(l)-k_{1} a_{1 y}(l)=æ a_{1}(l) w_{1}(l), \\
& k_{2} b_{2 y}(l)-k_{1} b_{1 y}(l)=æ b_{1}(l) w_{1}(l) .
\end{aligned}
$$

The first four conditions in (6) follow from the continuity of the velocity and temperature fields at the interface, and the fifth condition is the dynamic condition for tangential stresses. The last two conditions were obtained by taking into account relation (1) and the linear temperature dependence of the surface tension coefficient. From the condition for normal stresses we obtain that the interface remains flat. This assumption can be fulfilled, for example, under the action of sufficiently large capillary pressure [4]. 
Taking into account the no-slip conditions on the walls, vertical velocities $v_{j}(y)$ are excluded from the continuity equations

$$
v_{1}(y)=-\int_{0}^{y} w_{1}(z) d z, \quad 0 \leqslant y \leqslant l ; \quad v_{2}(y)=-\int_{y}^{h} w_{2}(z) d z, \quad l \leqslant y \leqslant h .
$$

Let us introduce dimensionless functions and parameters

$$
\begin{gathered}
W_{j}(\xi)=\frac{h^{2}}{\mathrm{M} \chi_{1}} w_{j}(y), \quad A_{j}(\xi)=\frac{a_{j}(y)}{a_{10}}, \quad F_{j}=\frac{h^{4}}{\mathrm{M} \chi_{1}^{2}} f_{j}, \quad \xi=\frac{y}{h}, \quad \mathrm{P}_{j}=\frac{\nu_{j}}{\chi_{j}}, \\
\mathrm{M}=\frac{æ a_{10} h^{3}}{\chi_{1} \mu_{2}}, \quad \mathrm{E}=\frac{\mathfrak{x}^{2} a_{10} h^{2}}{\mu_{2} k_{2}},
\end{gathered}
$$

where $\mathrm{P}_{j}$ are the Prandtl numbers, $\mathrm{M}$ is the Marangoni number. Parameters $\mathrm{M}$, E can be either positive or negative. Then we have the following inverse adjoint boundary value problem in dimensionless variables

$$
\begin{gathered}
W_{1 \xi \xi}=\frac{\mathrm{M}}{\mathrm{P}_{1}}\left[W_{1}^{2}-W_{1 \xi} \int_{0}^{\xi} W_{1}(z) d z\right]-\frac{F_{1}}{\mathrm{P}_{1}} \\
A_{1 \xi \xi}=\mathrm{M}\left[2 A_{1} W_{1}-A_{1 \xi} \int_{0}^{\xi} W_{1}(z) d z\right], \quad 0<\xi<\gamma \\
W_{2 \xi \xi}=\frac{\chi \mathrm{M}}{\mathrm{P}_{2}}\left[W_{2}^{2}-W_{2 \xi} \int_{1}^{\xi} W_{2}(z) d z\right]-\frac{\chi F_{2}}{\mathrm{P}_{2}}, \\
A_{2 \xi \xi}=\chi \mathrm{M}\left[2 A_{2} W_{2}-A_{2 \xi} \int_{1}^{\xi} W_{2}(z) d z\right], \quad \gamma<\xi<1,
\end{gathered}
$$

Boundary conditions for this problem follow from (5), (6) and (7)

$$
\begin{gathered}
W_{1}(0)=0, \quad W_{2}(1)=0, \quad A_{1}(0)=1, \quad A_{2}(1)=\frac{a_{20}}{a_{10}}=\delta, \\
W_{1}(\gamma)=W_{2}(\gamma), \quad A_{1}(\gamma)=A_{2}(\gamma), \\
W_{2 \xi}(\gamma)-\mu W_{1 \xi}(\gamma)=-2 A_{1}(\gamma), \\
A_{2 \xi}(\gamma)-k A_{1 \xi}(\gamma)=\mathrm{E} A_{1}(\gamma) W_{1}(\gamma), \\
\int_{0}^{\gamma} W_{1}(z) d z=0, \quad \int_{\gamma}^{1} W_{2}(z) d z=0,
\end{gathered}
$$

where $\gamma=l / h<1, \mu=\mu_{1} / \mu_{2}, \chi=\chi_{1} / \chi_{2}, k=k_{1} / k_{2}$. Integral conditions in (10) allow us to find unknown constants (pressure gradients along the layers) $F_{j}, j=1,2$. Functions $b_{j}(y)$ are found after solving problem (8)-(10) and they do not affect the velocity field in the layers.

Remark. Suppose that $|\mathrm{M}| \ll 1$ and we seek the solution of problem (8)-(10) in the form $W_{j}=W_{j}^{0}+\mathrm{M} W_{j}^{1}+\ldots, F_{j}=F_{j}^{0}+\mathrm{M} F_{j}^{1}+\ldots, A_{j}=A_{j}^{0}+\mathrm{M} A_{j}^{1}+\ldots$ Then, zeroth approximation has the form [5]

$$
\begin{gathered}
W_{1}^{0}(\xi)=\frac{\nu(1-\gamma)^{2}}{6 \gamma^{2} \mathrm{P}_{1}} F_{2}^{0}\left(-3 \xi^{2}+2 \gamma \xi\right), \quad A_{1}^{0}(\xi)=1+C_{1} \xi, \quad 0 \leqslant \xi \leqslant \gamma, \\
W_{2}^{0}(\xi)=\frac{\chi F_{2}^{0}}{6 \mathrm{P}_{2}}\left(-3 \xi^{2}+2(\gamma+2) \xi-1-2 \gamma\right), \quad A_{2}^{0}(\xi)=C_{2}(\xi-1)+\delta, \quad \gamma \leqslant \xi \leqslant 1, \\
-312-
\end{gathered}
$$




$$
C_{2}=\frac{1+\gamma C_{1}-\delta}{\gamma-1}, \quad F_{1}^{0}=\frac{\nu(1-\gamma)^{2}}{\gamma^{2}} F_{2}^{0}, \quad F_{2}^{0}=\frac{3 \gamma \mathrm{P}_{1} Z}{\nu(\gamma-1)(\gamma+\mu(1-\gamma))},
$$

and parameter $Z=1+\gamma C_{1}$ is the solution of the quadratic equation

$$
\frac{\gamma^{2}(1-\gamma)^{2} \mathrm{E}}{2(\gamma+\mu(1-\gamma))} Z^{2}+(\gamma+k(1-\gamma)) Z-(k(1-\gamma)+\gamma \delta)=0 .
$$

Expressions for dimensionless vertical speeds are determined with the use of (7). Simple calculations show that when $\mathrm{E}=0$ (no interfacial energy effect) there is unique solution of problem (8)-(10) for small Marangoni numbers.

\section{Numerical method and calculation results}

To solve problem (8)-(10) the Tau method is used. This method is a modification of the Galerkin method [6]. Let us introduce new variables: $\xi^{\prime}=\xi / \gamma$ for $j=1$ and $\xi^{\prime}=(1-\xi) /(1-\gamma)$ for $j=2$. Then problem (8)-(10) can be rewritten in the form (primes are omitted)

$$
\begin{gathered}
L_{1}\left(W_{1}, F_{1}\right) \equiv W_{1 \xi \xi}-\frac{\gamma^{2} \mathrm{M}}{\mathrm{P}_{1}}\left[W_{1}^{2}-W_{1 \xi} \int_{0}^{\gamma \xi} W_{1}(z) d z\right]-\frac{\gamma^{2} F_{1}}{\mathrm{P}_{1}}=0, \\
N_{1}\left(W_{1}, A_{1}\right) \equiv A_{1 \xi \xi}-\gamma^{2} \mathrm{M}\left[2 A_{1} W_{1}-A_{1 \xi} \int_{0}^{\gamma \xi} W_{1}(z) d z\right]=0, \quad 0<\xi<1, \\
L_{2}\left(W_{2}, F_{2}\right) \equiv W_{2 \xi \xi}-\frac{\chi(1-\gamma)^{2} \mathrm{M}}{\mathrm{P}_{2}}\left[W_{2}^{2}-W_{2 \xi} \int_{0}^{1-(1-\gamma) \xi} W_{2}(z) d z\right]-\frac{\chi(1-\gamma)^{2} F_{2}}{\mathrm{P}_{2}}=0, \\
N_{2}\left(W_{2}, A_{2}\right) \equiv A_{2 \xi \xi}-\chi(1-\gamma)^{2} \mathrm{M}\left[2 A_{2} W_{2}-A_{2 \xi} \int_{0}^{1-(1-\gamma) \xi} W_{2}(z) d z\right]=0, \quad 0<\xi<1, \\
W_{1}(0)=W_{2}(0)=0, \quad A_{1}(0)=1, \quad A_{2 \xi}(0)=0, \quad W_{1}(1)=W_{2}(1), \quad A_{1}(1)=A_{2}(1), \\
\frac{1}{1-\gamma} W_{2 \xi}(1)+\frac{\mu}{\gamma} W_{1 \xi}(1)=2 A_{1}(1), \quad \frac{1}{1-\gamma} A_{2 \xi}(1)+\frac{k}{\gamma} A_{1 \xi}(1)=-\mathrm{E} A_{1}(1) W_{1}(1), \\
\int_{0}^{1} W_{1}(z) d z=0, \quad \int_{0}^{1} W_{2}(z) d z=0 .
\end{gathered}
$$

An approximate solution of problem (12)-(16) is sought in the following form

$$
W_{j n}(\xi)=\sum_{k=1}^{n} W_{j}^{k} R_{k}(\xi), \quad A_{j n}(\xi)=\sum_{k=0}^{n} A_{j}^{k} R_{k}(\xi)
$$

where $R_{k}(z)=P_{k}(2 z-1)$ are shifted Legendre polynomials, $z \in[0,1], P_{k}(z)$ are ordinary Legendre polynomials [7]. Taken into account orthogonality of the Legendre polynomials $R_{k}(z)$ on the interval $[0,1]$

$$
\int_{0}^{1} R_{k}(z) R_{m}(z) d z=\delta_{k m} h_{m}, \quad h_{m}=\frac{1}{2 m+1}, \quad \delta_{k m}= \begin{cases}1, & k=m, \\ 0, & k \neq m\end{cases}
$$


we obtain from integral conditions (16) that $W_{1}^{0}=W_{2}^{0}=0$. Coefficients $W_{j}^{k}, A_{j}^{k}$ and constants $F_{1}, F_{2}$ are determined from the system of Galerkin approximations

$$
\int_{0}^{1} L_{j}\left(W_{j n}, F_{j}\right) R_{m}(\xi) d \xi=0, \quad \int_{0}^{1} N_{j}\left(W_{j n}, A_{j n}\right) R_{m}(\xi) d \xi=0, \quad m=0, \ldots, n-2, \quad j=1,2
$$

and transformed boundary conditions (14)-(15)

$$
\begin{gathered}
\sum_{k=1}^{n}(-1)^{k} W_{1}^{k}=0, \quad \sum_{k=1}^{n}(-1)^{k} W_{2}^{k}=0, \quad \sum_{k=0}^{n}(-1)^{k} A_{1}^{k}=1, \quad \sum_{k=0}^{n} A_{2}^{k} R_{k}^{\prime}(0)=0, \\
\sum_{k=1}^{n} W_{1}^{k}=\sum_{k=1}^{n} W_{2}^{k}, \quad \sum_{k=0}^{n} A_{1}^{k}=\sum_{k=0}^{n} A_{2}^{k}, \\
\frac{1}{1-\gamma} \sum_{k=1}^{n} W_{2}^{k} R_{k}^{\prime}(1)+\frac{\mu}{\gamma} \sum_{k=1}^{n} W_{1}^{k} R_{k}^{\prime}(1)=2 \sum_{k=0}^{n} A_{1}^{k}, \\
\frac{1}{1-\gamma} \sum_{k=0}^{n} A_{2}^{k} R_{k}^{\prime}(1)+\frac{k}{\gamma} \sum_{k=0}^{n} A_{1}^{k} R_{k}^{\prime}(1)=-\mathrm{E} \sum_{k=0}^{n} A_{1}^{k} \sum_{k=1}^{n} W_{1}^{k} .
\end{gathered}
$$

We also taken into account that $R_{k}(1)=1, R_{k}(0)=(-1)^{k}$. Thus, equations (18), (19) form a closed system of algebraic nonlinear equations for coefficients $W_{j}^{k}, A_{j}^{k}$ and constants $F_{j}, j=1,2$.

Calculations were carried out for parameters of water $(j=1)$ and water vapor $(j=2)$ system on the saturation line at temperature $300^{\circ} \mathrm{C}: \rho_{1}=712 \mathrm{~kg} / \mathrm{m}^{3}, \rho_{2}=46.8 \mathrm{~kg} / \mathrm{m}^{3}, \nu_{1}=$ $=1.3 \cdot 10^{-7} \mathrm{~m}^{2} / \mathrm{s}, \quad \nu_{2}=4.2 \cdot 10^{-7} \mathrm{~m}^{2} / \mathrm{s}, \quad \chi_{1}=1.4 \cdot 10^{-7} \mathrm{~m}^{2} / \mathrm{s}, \quad \chi_{2}=2.5 \cdot 10^{-7} \mathrm{~m}^{2} / \mathrm{s}, \quad k_{1}=$ $=0.54 \mathrm{~W} / \mathrm{m} \cdot{ }^{\circ} C, k_{2}=0.0719 \mathrm{~W} / \mathrm{m} \cdot{ }^{\circ} C, æ=1.4 \cdot 10^{-4} \mathrm{~N} / \mathrm{m} \cdot{ }^{\circ} C, \mathrm{P}_{1}=0.96, \mathrm{P}_{2}=1.69, \mathrm{E}=0.6$, $\mathrm{M}=16.5, \delta=1\left(a_{10}=a_{20}\right), h=1 \cdot 10^{-9} \mathrm{~m}, l=0.5 \cdot 10^{-9} \mathrm{~m}$ and $n=15$. Three different values of dimensionless constants $F_{1}, F_{2}$ were obtained: $\left\{F_{1}^{1}=-0.998, F_{2}^{1}=-3.4\right\},\left\{F_{1}^{2}=26.241\right.$, $\left.F_{2}^{2}=82.04\right\}$ and $\left\{F_{1}^{3}=-68.86, F_{2}^{3}=-352.18\right\}$ (superscript indicates the solution number). The obtained values at $n=15$ and at $n=16$ differ by $10^{-20}, 10^{-12}$ and $10^{-7}$ for $F^{1}, F^{2}$ and $F^{3}$ respectively. It means good convergence of the Tau method in solving this boundary value problem. Fig. 1 shows the profiles of dimensionless functions $W_{j}(\xi)$ and transverse velocities $V_{j}(\xi)$ for values $F^{1}$ and $F^{2}$, respectively. Here functions $W(\xi)$ and $V(\xi)$ coincide with functions $W_{j}(\xi)$ and $V_{j}(\xi), j=1,2$ on their domains of definition. Profiles $W(\xi)$ and $V(\xi)$ qualitatively coincide for $F^{3}$ and $F^{1}$. However, the flow is more intense for $F^{3}$ because $\max _{\xi \in[0,1]}\left|V\left(\xi, F^{3}\right)\right|=0.365$, $\max _{\xi \in[0,1]}\left|W\left(\xi, F^{3}\right)\right|=6.04$, and $\max _{\xi \in[0,1]}\left|V\left(\xi, F^{1}\right)\right|=0.0032, \max _{\xi \in[0,1]}\left|W\left(\xi, F^{1}\right)\right|=0.044$.

Fig. 2 shows the relationship between dimensionless functions $W_{j}(\xi)$ and transverse velocities $V_{j}(\xi)$ for $F^{1}$ and dimensionless parameter $\delta$. It is seen that with decreasing $\delta$ the flow intensity decreases. For $F^{2}$ and $F^{3}$ the situation is similar.

One should note that dimensionless parameters $\mathrm{E}, \mathrm{M}, \mathrm{P}_{1}, \mathrm{P}_{2}$ affect only the intensity of flows. For example, for $F^{1}$ with decreasing number $\mathrm{E}$ the flow rate increases and with increasing Prandtl number $\mathrm{P}_{1}$ the flow rate decreases. It was also established that with decreasing Marangoni number $\mathrm{M}$ the obtained solutions $F^{1}$ and $F^{2}$ tend to solutions of the model problem $F_{1}^{01}=$ $=-1.036, F_{2}^{01}=-3.506$ and $F_{1}^{02}=654.63, F_{2}^{02}=2215.12$, respectively (see (11)). For example, at $\mathrm{M}=0.001$ we obtain $\left|F_{j}^{01}-F_{j}^{1}\right| \approx 10^{-9}$ and $\left|F_{j}^{02}-F_{j}^{2}\right| \approx 10^{-3}, j=1,2$.

This research was supported by the Russian Foundation for Basic Research (grant 17-0100229). 

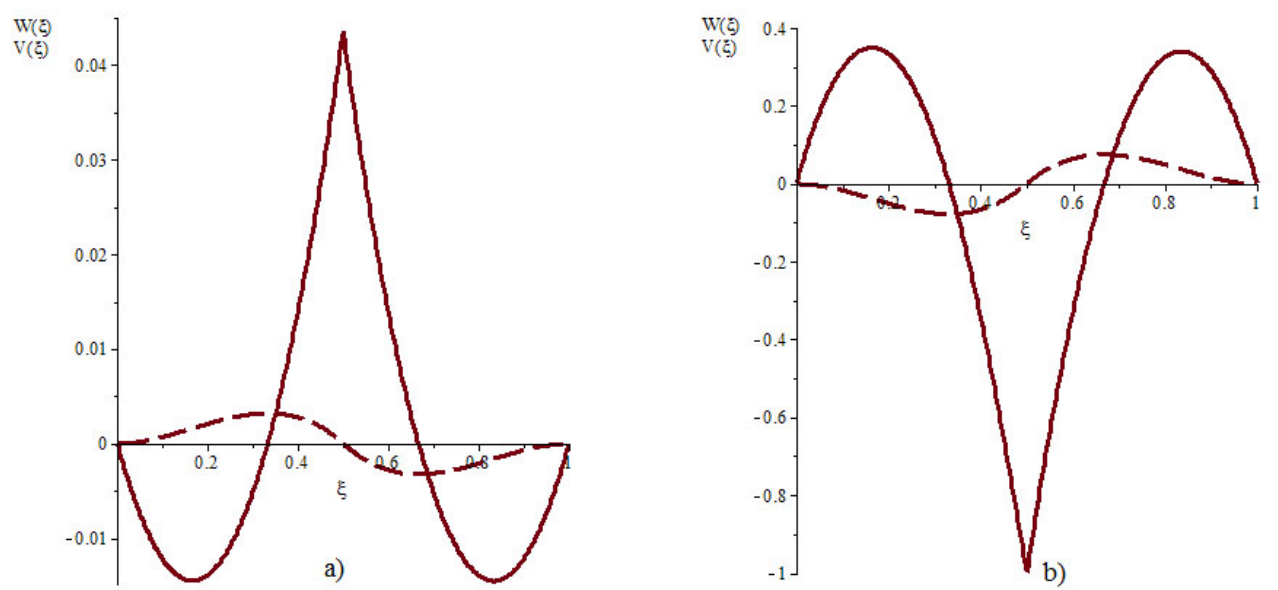

Fig. 1. Profiles of dimensionless functions $W(\xi)(-)$ and transverse velocities $V(\xi)(--)$ for $F^{1}(\mathrm{a})$ and $F^{2}(\mathrm{~b})$
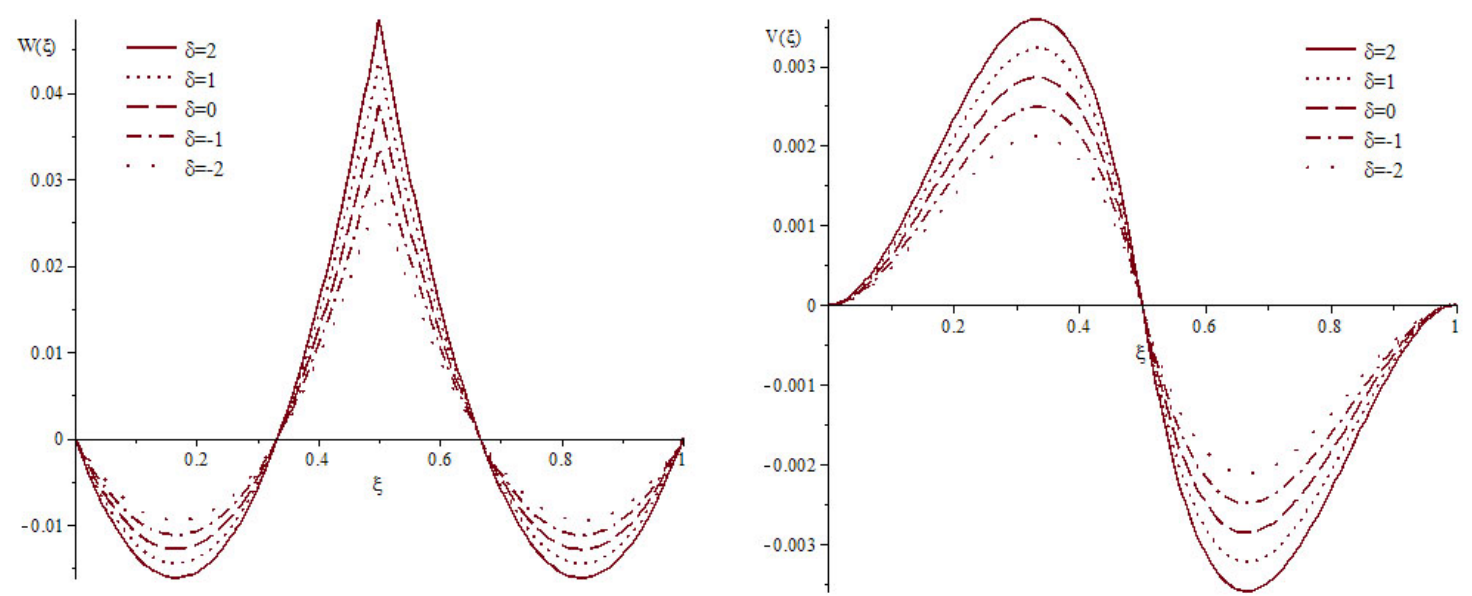

Fig. 2. Profiles of dimensionless functions $W(\xi)$ and transverse velocities $V(\xi)$ for $F^{1}$

\section{References}

[1] V.K.Andreev, V.E.Zahvataev, E.A.Ryabitskii, Thermocapillary Instability, Nauka, Sibirskoe otdelenie, Novosibirsk, 2000 (in Russian).

[2] F.E.Torres, E.Helborzheimer, Temperature gradients and drag effects produced by convection of interfacial internal energy around bubbles, Phys. Fluids A, 5(1993), no. 3, 537-549.

[3] K.Hiemenz, Die Grenzschicht an einem in den gleichförmigen Flüssigkeitsstrom eingetauchten geraden Kreiszylinder, Dinglers Poliytech. J., 326(1911), 321-440.

[4] R.Kh.Zeytounian,The Benard-Marangoni thermocapillary-instability problem, 41(1998), no. $3,241-267$. 
[5] V.K.Andreev, The solutions properties of conjugated nonlinear boundary value problem describing a stationary flow of a two liquids in the channel, Some actual problems of modern mathematics and mathematical education. Herzen readings, Scientific conference materials, 2018, 18-23.

[6] C.A.J.Fletcher, Computational Galerkin method, Springer-Verlag, 1984.

[7] Gabor Szego, Orthogonal polynomials American Mathematical Society Colloquium Publications, Vol. 23, Revised ed. American Mathematical Society, Providence, R.I., 1959.

\title{
Двумерное плоское термокапиллярное течение двух несмешивающихся жидкостей
}

Елена Н. Лемешкова

Институт вычислительного моделирования СО РАН Академгородок, 50/44, Красноярск, 660036

Россия

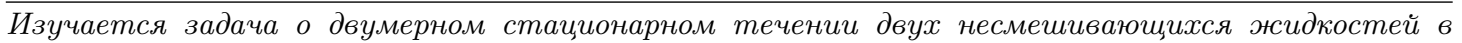
плоском канале. На твердых стенках канал поддерживает заданное распределение температуры. ЖКидкости контактируют через общую поверхность раздела, на которой учитываются затраты энергии на ее деформащию. Температура в жидкостях распределена по квадратичному закону, что согласуется с полем скоростей типа Хименца. Математический анализ такого течения приводит $\kappa$ возникновению сопряэенной краевой задачи, которая является нелинейной и обратной относительно градиентов давлений вдоль канала. Применение к ней тау-метода показывает, что она имеет три различных решения. Численно установлено, что полученные решения с уменъшением числа Марангони сходятся к решениям задачи о ползущем течении. Для кажсого из решений построены характерные структуры течения.
\end{abstract}

Ключевые слова: граница раздела, термокапиллярность, обратная задача, тау-метод. 